Original Article

\title{
DEVELOPMENT AND VALIDATION OF A HPTLC METHOD FOR SIMULTANEOUS DENSITOMETRIC ANALYSIS OF GLYCYRRHETIC ACID AND SOLASODINE IN HERBAL DRUG FORMULATION
}

\author{
KINJAL PATEL*, CHAITANYA BHATT \\ Department of Pharmacognosy, Shree Swaminarayan Sanskar Pharmacy College, Zundal Ghandhinagar, India \\ Email: kinjal9387@gmail.com
}

Received: 27 Jan 2017, Revised and Accepted: 20 Apr 2017

\begin{abstract}
Objective: To develop and validate a simple, precise, selective, and accurate high-performance thin layer chromatographic method for simultaneous densitometric analysis of glycyrrhetic acid and solasodine in the polyherbal formulation.

Methods: The method was developed using HPTLC silica gel GF 254 precoated aluminium plate as the stationary phase and Chloroform: Methanol $(9: 1 \mathrm{v} / \mathrm{v})$ as the mobile phase. Quantization of glycyrrhetic acid was achieved by determining the area under the curve at $267 \mathrm{~nm}$ using CAMAG TLC Scanner and CATS 3 software. Since the structure of solasodine lacks conjugated double bond, it does not give any fluorescence either in the absorbance mode or reflectance mode hence solasodine was derivatized using $0.5 \%$ anisaldehyde sulphuric acids which gave a bluish spot as seen on TLC plate. These spots were scanned at $546 \mathrm{~nm}$ wavelength using CAMAG TLC Scanner and CATS 3 software.
\end{abstract}

Results: The retention factor for glycyrrhetic acid and solasodine were found to be $0.52 \pm 0.01,0.40 \pm 0.01 \% \mathrm{w} / \mathrm{w}$ respectively. The developed HPTLC method was validated using parameters described in International Conference on Harmonization (ICH) guideline. The proposed method showed good linearity in the range of 400-2000 ng spot ${ }^{-1}$ for glycyrrhetic acid as well as for solasodine. The content of glycyrrhetic acid and solasodine in marketed polyherbal formulation were found to be $0.67 \% \pm 0.8$ and $0.10 \pm 0.35 \% \mathrm{w} / \mathrm{w}$ respectively.

Conclusion: The developed method can be used as quality control tool for the routine analysis of glycyrrhetic acid and solasodine in polyherbal formulation.

Keywords: Glycyrrhetic acid, Solasodine, HPTLC, Simultaneous estimation

(c) 2017 The Authors. Published by Innovare Academic Sciences Pvt Ltd. This is an open access article under the CC BY license (http://creativecommons.org/licenses/by/4.0/) DOI: http://dx.doi.org/10.22159/ijcpr.2017v9i4.20752

\section{INTRODUCTION}

Glycyrrhetic acid is an aglycone of glycyrrhizin, a triterpenoid compound isolated from Glycyrrhizaglabra. It possesses various activities like antiinflammatory, analgesic, antiasthmatic etc [1, 2]. Solasodine is a steroidal glycoalkaloid, isolated from Solanum xanthocarpum possesses antiasthmatic and mucolytic activities [3, 4], hence they forms one of the most important constituents of almost each and every polyherbal formulation used for the management of asthmatic conditions. Extensive literature survey revealed that few HPTLC [5-7] and HPLC [8,9] methods are available for estimation of Glycyrrhetic acid individually and in combination with other marker compounds in the same plant. HPTLC $[10,11]$ and HPLC $[12,13]$ methods have also been reported for estimation of Glycyrrhetic acid individually and in combination with other marker compounds. Even though both of these compounds are most commonly used together, there were no reports found for simultaneous estimation of glycyrrhetic acid and solasodine. Hence the objective of the work was to develop and validate a simple, accurate and reproducible method for the simultaneous HPTLC analysis of glycyrrhetic acid and solasodine in polyherbal formulations.

\section{MATERIALS AND METHODS}

Solvents and chemicals

Standard of glycyrrhetic acid and solasodine were purchased from Natural Remedies Private limited, Bangalore, India. Polyherbal formulation (Askon syrup) used in the study was purchased from the local market of Ahmedabad. All chemicals and reagents used were of analytical reagent grade and purchased from Merck specialities Pvt. Ltd. (Mumbai, India). Double distilled water was used in the present work.

\section{Instrumentation and chromatographic conditions}

The sample solutions were spotted on precoated silica gel aluminium plate $60 \mathrm{~F} 254(20 \mathrm{~cm} \times 10 \mathrm{~cm})$ with $250 \mu \mathrm{m}$ thickness (E. Merck, Darmstadt, Germany) in the form of bands of $6 \mathrm{~mm}$ width with a Hamilton syringe $(100 \mu \mathrm{L})$ using a Camag Linomat V
(Switzerland) sample applicator. The plates were prewashed with methanol, activated in an oven at $105{ }^{\circ} \mathrm{C}$ for $20 \mathrm{~min}$, and then allowed to cool to attain room temperature before sample application. The slit dimension was kept at $5 \mathrm{~mm} \times 0.45 \mathrm{~mm}$ and 10 $\mathrm{mm} / \mathrm{s}$ scanning speed was employed. Plates were then developed, at a constant temperature with $20 \mathrm{ml}$ mobile phase consisting of Chloroform: Methanol $(9: 1 \mathrm{v} / \mathrm{v})$. Linear ascending development was carried out in $20 \mathrm{~cm} \times 10 \mathrm{~cm}$ twin trough glass chamber (Camag, Muttenz, Switzerland) previously saturated with the mobile phase. The optimized chamber saturation time for mobile phase was 25 min at room temperature $\left(25 \pm 2{ }^{\circ} \mathrm{C}\right)$ at a relative humidity of $60 \pm 5 \%$. The optimum wavelength for detection and quantification of glycyrrhetic acid was $267 \mathrm{~nm}$. Since solasodine lacks any conjugated double bond it does not give any fluorescence either in the absorbance mode or reflectance mode so it required ion-pair complexation of the solasodine followed by in situ colour development in an acidic solvent front for achieving a chromophore which could be scanned in the visible range of the scanner. Densitometric scanning was performed within $10 \mathrm{~min}$ after derivatization process using Camag TLC scanner III with winCATS software version 1.3.4.

\section{Preparation of standard solutions}

\section{Preparation of standard solution of glycyrrhetic acid}

A stock solution of glycyrrhetic acid (1 mg ml-1) was prepared by dissolving $10 \mathrm{mg}$ of accurately weighed glycyrrhetic acid in chloroform and volume was made up to $10 \mathrm{ml}$ with chloroform in a volumetric flask. From this, $1.0 \mathrm{ml}$ of stock solution was transferred to $10 \mathrm{ml}$ volumetric flask and the volume was adjusted to $10 \mathrm{ml}$ with chloroform to obtain a standard solution containing $100 \mu \mathrm{g} \mathrm{ml}-1$ of glycyrrhetic acid.

\section{Preparation of standard solution of solasodine}

A stock solution of solasodine $\left(1 \mathrm{mg} \mathrm{ml}^{-1}\right)$ was prepared by dissolving $100 \mathrm{mg}$ of accurately weighed solasodine in methanol and 
the volume was made up to $100 \mathrm{ml}$ with methanol. From this $1.0 \mathrm{ml}$ of stock solution was transferred to $10 \mathrm{ml}$ volumetric flask and the volume was adjusted to $10 \mathrm{ml}$ with methanol to obtain a standard solution containing $100 \mu \mathrm{g} \mathrm{ml}^{-1}$ of solasodine.

\section{Selection of detection wavelength}

After chromatographic development and derivatization process, bands were scanned over the range of 400-2000 ng spot ${ }^{-1}$ and the spectra were overlain. The one marker of the ingredients of the formulation is glycyrrhetic acid which does not require any derivatization as they have distinct absorption maxima under UV. Hence, solasodine was derivatized with $0.5 \%$ anisaldehyde sulphuric acid which gave a bluish spot as seen from the chromatogram. These spots were scanned at $546 \mathrm{~nm}$ wavelength, at which the best detector response was obtained.

\section{Construction of calibration plots}

Linearity was evaluated in the range of 400-2000ng spot ${ }^{-1}$ for glycyrrhetic acid and solasodine. For the calibration curve accurately measured a standard stock solution of glycyrrhetic acid and solasodine $(400,800,1200,1600,2000 \mathrm{ng})$ were spotted on precoated TLC plate under nitrogen stream using Linomat V spotter. Then chromatographed and scanned as described above.

\section{Sample preparation for glycyrrhetic acid and solasodine}

$40 \mathrm{ml}$ of syrup was extracted with $60 \mathrm{ml}$ chloroform for $20 \mathrm{~min}$. Then the solution was filtered and the filtrate was discarded. Then the marc was refluxed for $1 \mathrm{~h}$ with $90 \mathrm{ml}$ of $0.5 \mathrm{M} \mathrm{H}_{2} \mathrm{SO}_{4}$. Then the solution was cooled and filtered. The unfiltered mixture was shaken with chloroform $(2 \times 20 \mathrm{ml})$ to extract glycyrrhetic acid. The filtrate was basified with ammonia and extracted with chloroform $(2 \times 20$ $\mathrm{ml}$ ) to extract solasodine. Both the Chloroform extracts were combined. Volume was made up to $100 \mathrm{ml}$ with chloroform.

\section{Assay validation}

The proposed method was optimized and validated as per the International Conference on Harmonization (ICH) guidelines [14]. All measurements were performed in triplicates.

\section{Precision studies}

In order to judge the quality of the proposed HPTLC method, precision was determined. The precision of the proposed HPTLC method was verified by Intra-day and inter-day precision studies. Intra-day precision was performed by analysis of single concentration in six replicates of mixed standard solutions of glycyrrhetic acid (1200ng band $\left.{ }^{-1}\right)$, solasodine (1200ng band $\left.{ }^{-1}\right)$ which were prepared on the same day. Intermediate precision was performed by repeating analysis on three consecutive days. The peak areas were recorded and percentage relative standard deviation (\% RSD) was calculated.

\section{Accuracy}

In order to evaluate the validity of the proposed method, accuracy was evaluated through the percentage recoveries of known amounts of a mixture of glycyrrhetic acid and solasodine added to solutions of marketed herbal formulation. Marketed herbal formulation was spiked with the known amount of standards, and the percent ratios between the recovered and expected concentrations were calculated. The analyzed samples were spiked with 80, 100 and 120 $\%$ of $0.512 \mathrm{mg}$ glycyrrhetic acid and $4.05 \mathrm{mg}$ of solasodine standard solutions. The percent ratios between the recovered and expected concentrations were estimated.

\section{Specificity}

The specificity of the proposed HPTLC method was estimated by analyzing the standard marker and sample. Peaks for glycyrrhetic acid and solasodine were confirmed by comparing the retention time. Excipients present in the herbal formulation did not interfere with the peaks of glycyrrhetic acid and solasodine. The method was found to be specific.

\section{Robustness studies}

The effects of small, deliberate variation of the analytical conditions on the peak areas of the drugs were examined. The robustness of the proposed chromatographic method was performed at a concentration of $1200 \mathrm{ng} \mathrm{band}^{-1}$ for glycyrrhetic acid as well as for solasodine. The standard deviation of peak areas and \% RSD were calculated for each variable parameter.

\section{RESULTS AND DISCUSSION \\ HPTLC method optimization}

For HPTLC method, Chromatographic conditions were optimized to achieve the best resolution and peak shape for glycyrrhetic acid and solasodine. Different mobile phases in different proportion were tried and the mobile phase containing Chloroform: Methanol (9:1 $\mathrm{v} / \mathrm{v}$ ) was selected as optimal for obtaining well-resolved peaks of glycyrrhetic acid $\left(\mathrm{R}_{\mathrm{f}}=0.52\right)$ and solasodine $\left(\mathrm{R}_{\mathrm{f}}=0.40\right)$ with acceptable system suitability parameters. The optimum wavelength for detection and quantification of glycyrrhetic acid was $267 \mathrm{~nm}$ and solasodine was derivatized with $0.5 \%$ anisaldehyde sulphuric acid which gave a bluish spot as seen from the chromatogram. These spots were scanned at $546 \mathrm{~nm}$ wavelength. The retention factors for glycyrrhetic acid and solasodine were found to be $0.52 \pm 0.01$ and $0.40 \pm 0.01$, respectively. Representative TLC chromatogram obtained from a mixed standard solution of glycyrrhetic acid and solasodine is shown in fig. 1 and 2 .

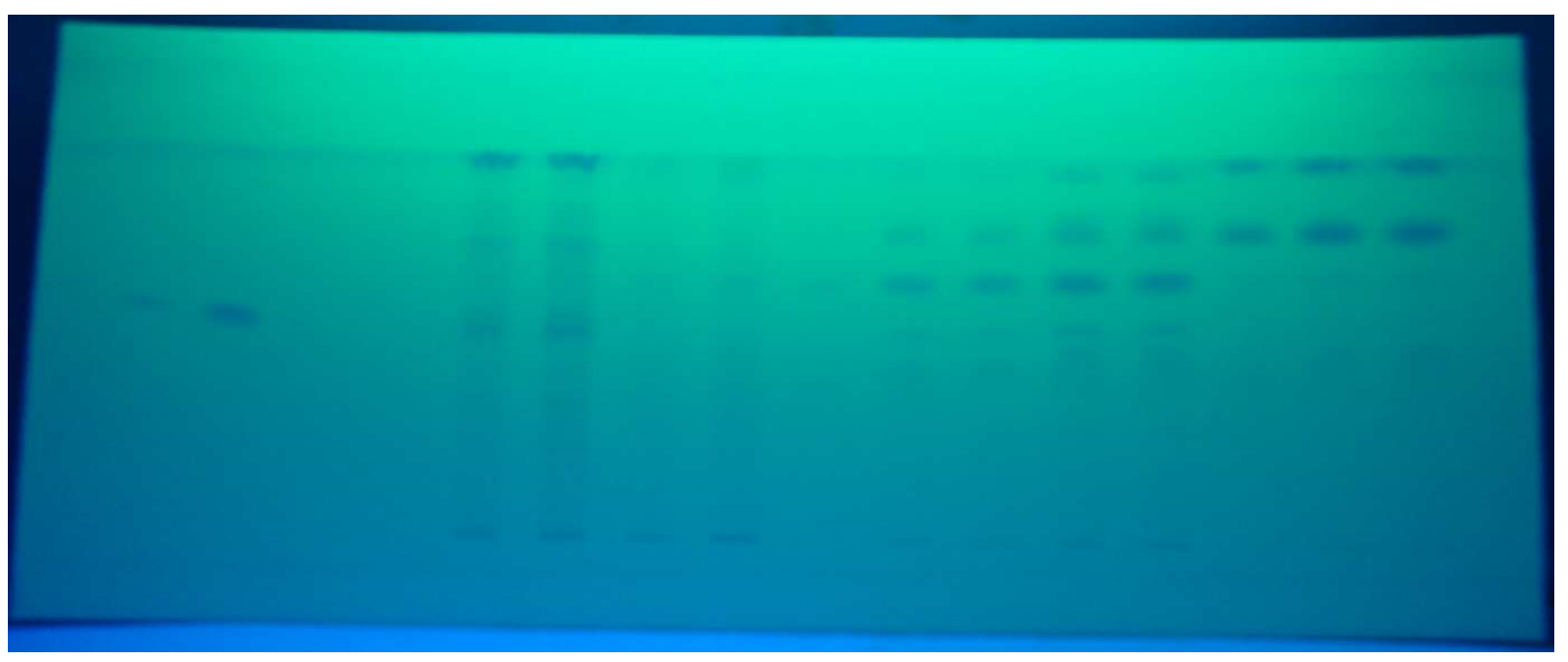

Fig. 1: TLC chromatogram of glycyrrhetic acid at $254 \mathrm{~nm}$ 


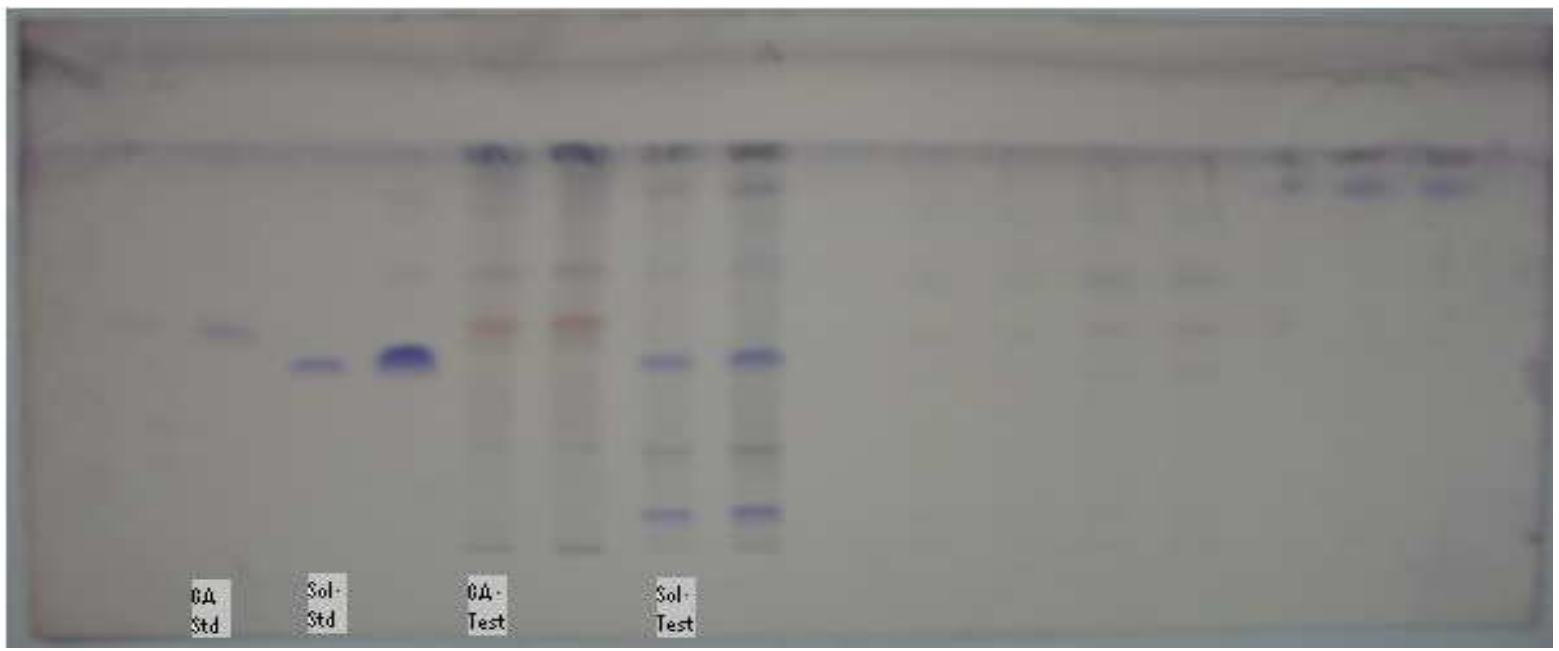

Fig. 2: TLC chromatogram of glycyrrhetic acid and solasodine after derivatization

\section{Linearity, limit of detection and quantitation}

The results were found to be linear in a range of 400-2000 ng spot s $^{-1}$ for glycyrrhetic acid and solasodine. The correlation coefficients (r) for the plots were 0.993 for glycyrrhetic acid and 0.992 for solasodine. The calibration plots obtained for glycyrrhetic acid and solasodine are shown in fig. 3 and fig. 4. The LOD and LOQ for glycyrrhetic acid and solasodine were found to be 22.97 and $23.99 \mathrm{ng}$ and 69.61and $72.72 \mathrm{ng}$, respectively.

\section{Precision}

The developed HPTLC method was found to be precise (table 1), with \% RSD values for repeatability and intermediate precision studies below $2 \%$ as recommended by ICH Q2 (R1) guideline.

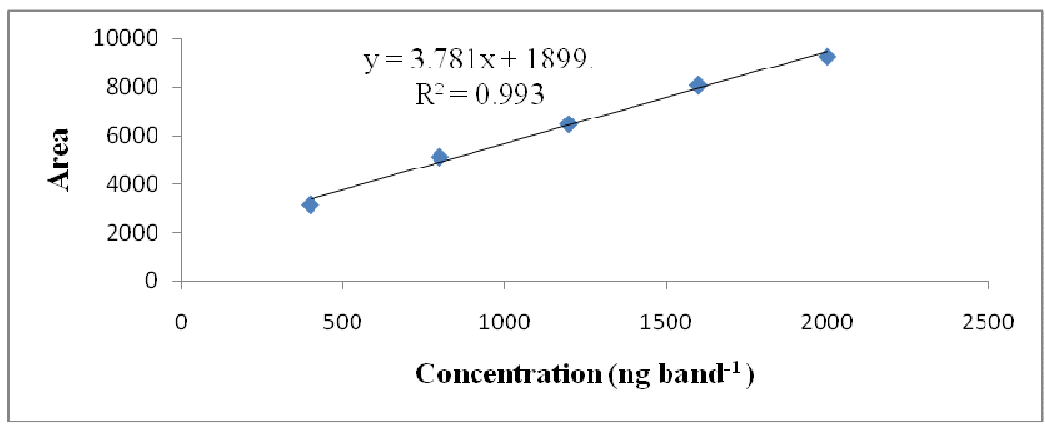

Fig. 3: Calibration curve for glycyrrhetic acid

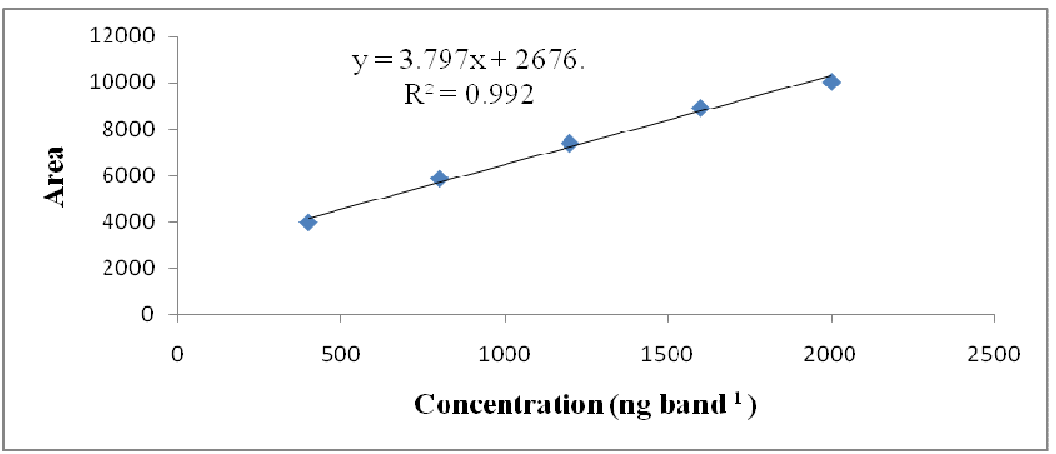

Fig. 4: Calibration curve for solasodine

Table 1: Intra and inter-day precision of the HPTLC method $(n=6)$

\begin{tabular}{llll}
\hline Marker compounds & Actual concentration & Intra-day variation, as RSD (\%) & Inter-day variation, as RSD (\%) \\
\hline Glycyrrhetic acid & 1200 & 0.41 & 0.73 \\
Solasodine & 1200 & 0.38 & 0.94 \\
\hline
\end{tabular}

$\mathrm{RSD}=$ Relative standard deviation 


\section{Accuracy}

Satisfactory recoveries for Glycyrrhetic acid and Solasodine were obtained (table 2), which indicate that the proposed chromatographic method is reliable for the simultaneous quantification of selected markers in this herbal formulation.

\section{Analysis of marketed herbal formulation}

The validity of the proposed method was applied to standardization for herbal dosage forms viz. Kofol OR AskonSyrup. The shape of the peaks was not altered by other substances present in the matrix. The percent content of both viz., Glycyrrhetic acid and Solasodine in marketed herbal formulation was found to be $0.67 \% \pm 0.8$ and $0.10 \pm 0.35 \%$ respectively.

\section{Robustness studies}

Robustness of the method checked after deliberate alterations of the analytical parameters showed that areas of peaks of interest and retention factor remained unaffected by small changes of the operational parameters $(\% \mathrm{RSD}<2)$. The summary of validation parameters of proposed method are given in table 3.

Table 2: Results of recovery studies $(n=3)$

\begin{tabular}{|c|c|c|c|c|}
\hline Drug & Amount of sample (ng) & Amount of standard added(ng) & $\begin{array}{l}\text { Amount of standard } \\
\text { recovered } \pm S D\end{array}$ & $\%$ recovery \pm C. V. (\%) \\
\hline \multirow{3}{*}{ Glycyrrhetic acid } & 640 & 512 & $505.65 \pm 0.06$ & $98.76 \pm 0.01 \%$ \\
\hline & 640 & 640 & $642.49 \pm 0.13$ & $100.39 \pm 0.03 \%$ \\
\hline & 640 & 768 & $762.67 \pm 1.10$ & $99.31 \pm 0.15 \%$ \\
\hline \multirow[t]{3}{*}{ Solasodine } & 506 & 405 & $402.4 \pm 1.9$ & $99.36 \pm 0.47 \%$ \\
\hline & 506 & 506 & $500.4 \pm 0.51$ & $98.89 \pm 0.10 \%$ \\
\hline & 506 & 607 & $609.7 \pm 1.96$ & $100.44 \pm 0.33 \%$ \\
\hline
\end{tabular}

Table 3: Summary of validation parameters of proposed method

\begin{tabular}{lll}
\hline Parameters & Glycyrrhetic acid & Solasodine \\
\hline Linearity range(ng spot-1) & $400-2000$ & $400-2000$ \\
Correlation coeficient & 0.993 \\
Precision & & 0.992 \\
Reproducibility (R. SD) & 0.16 \\
Instrumental (n=6) & 0.41 \\
Intraday (n= 6) & 0.73 \\
Inter day (n=6) & \\
Accuracy (\% Recovery) & $99.49 \%$ \\
Limit of detection & $22.97 \mathrm{ng}$ \\
Limit of quantification & $69.61 \mathrm{ng}$ & 0.38 \\
Specificity & Specific & 0.94 \\
\hline
\end{tabular}

LOD =Limit of detection.,LOQ =Limit of quantitation., $\mathrm{RSD}=$ Relativestandard deviation., $\mathrm{n}=$ Number of determinations

\section{CONCLUSION}

The validated HPTLC method employed proved to be simple, fast, accurate, precise and robust and thus can be useful in the quality control of glycyrrhetic acid and solasodine in the polyherbal formulation.

\section{CONFLICT OF INTERESTS}

Declare none

\section{REFERENCES}

1. Tolstikov GA, Baltina LA, Serdyuk NG. Glycyrrhetic acid: a review. Pharm Chem J 1998;32:402-12.

2. Ram A, Bhattacharya I, Das M, Ghosh B, Gangal SV. Evaluation of glycyrrhizin, a compound isolated from Glycyrrhiza glabra for the anti-asthmatic activity using a mouse model of asthma. Indian J Aerobiol 2001;14:42.

3. Subharani K. Chemical constituents and medicinal properties of Solanumxanthocarpum: a review. Int J Sci Res 2016;5:824-6.

4. Bector NP, Puri AS. Solanum Xanthocarpum (Kantakari) in chronic bronchitis, bronchial asthma and non-specific unproductive cough. J Assoc Physicians India 1971;19:741-4.

5. Rathee P, Rathee S, Ahuja D. Simultaneous quantification of glycyrrhetinic acid and apigenin using HPTLC from Glycyrrhiza glabra Linn. Eurasian J Anal Chem 2010;5:95-103.

6. Trivedi A, Mishra SH. A simple and rapid method for simultaneous estimation of glycyrrhetinic acid and piperine by HPTLC in the herbomineral formulation. J Adv Pharm Tech Res 2010;1:190-8.

7. Vampa G, Benvenuti S. Separation of $18 \alpha$-and 18ß-glycyrrhetinic acid by high-performance thin-layer chromatographic densitometry. J Chromatography 1991;543:479-82.
8. Esmaeili S, Naghibi F, Mosaddegh M, Nader N. Determination of 18 ß-glycyrrhetinic acids in Glycyrrhiza glabra L. extract by HPLC. Iranian J Pharm Res 2006;5:137-41.

9. Zhou S, Zhang X, Duan X, Mei H. HPLC determination of glycyrrhizic acid andglycyrrhetinic acid in weiyanning granules. Biomed Res 2015;26:311-4.

10. Shanker K, Gupta S, Shrivastava P, Shrivastava SK, Sing SC, Gupta MM. Simultaneous determination of three steroidal glycoalkaloids in Solanum xanthocarpum by high-performance thin layer chromatography. J Pharma Biomed Anal 2011;54:497-502.

11. Trivedi P, Pundarikakshudu K. Novel TLC densitometric method for quantification of solasodine in various Solanum species, market samples and formulations. Chromatogr 2006;65:239-43.

12. Kittipongpatana N, Porter JR, Hock RS. An improved high performance liquid chromatographic method for the quantification of solasodine. Phytochem Anal 1999;10:26-31.

13. Trivedi PD, Patel BC, Rathnam SB, Pundarikakshudu K. A validated a liquid chromatographic method for estimation of solasodine in various Solanum species and market formulations. J AOAC Int 2006;89:1519-23.

14. ICH Harmonised Tripartite Guideline, Validation of Analytical Procedures: Text and Methodology Q2 (R1), Nov; 2005.

\section{How to cite this article}

- $\quad$ Kinjal Patel, Chaitanya Bhatt. Development and validation of a HPTLC method for simultaneous densitometric analysis of glycyrrhetic acid and solasodine in herbal drug formulation. Int $\mathrm{J}$ Curr Pharm Res 2017;9(4):29-32. 